\title{
Differences between palpation and static/dynamic tests to diagnose painful temporomandibular disorders in patients with Lyme disease
}

\author{
Magdalena Osiewicz ${ }^{1}$ (D) $\cdot$ Daniele Manfredini $^{2} \cdot$ Grażyna Biesiada $^{3} \cdot$ Jacek Czepiel $^{3} \cdot$ Aleksander Garlicki $^{3}$. \\ Jolanta Pytko-Polończyk ${ }^{1}$. Frank Lobbezoo ${ }^{4}$
}

Received: 7 September 2018 / Accepted: 2 April 2019 / Published online: 13 April 2019

(C) The Author(s) 2019

\begin{abstract}
Objectives The aim was to determine the frequency of Research Diagnostic Criteria for Temporomandibular Disorders (RDC/ TMD)-based pain diagnoses and dynamic/static tests-based pain diagnoses, and to assess the agreement of palpation tests with static/dynamic tests.

Materials and methods Eighty-six $(N=86)$ adult patients with Lyme disease (mean age $57.0 \pm 14.3$ years; male/female ratio was 42/44) were examined according to techniques described in the RDC/TMD. Additionally, dynamic/static tests were performed. For RDC/TMD-based pain diagnoses and dynamic/static tests-based pain diagnoses, descriptive frequencies were calculated. Differences between the frequency of palpation-based diagnoses and of dynamic/static-based diagnoses as well as the agreement between pain diagnoses established with the two diagnostic approaches were assessed.

Results RDC/TMD-based pain diagnoses were made in 61 patients for myofascial pain and in 11 patients for arthralgia and/or osteoarthritis. Based on dynamic/static tests, mainly myogenous pain was diagnosed in 6 patients, and a mainly arthrogenous pain in 5. The agreement of palpation tests with static/dynamic tests in Lyme disease population was poor.

Conclusion A high prevalence of TMD symptoms was found in patients with Lyme disease. The results suggest that using palpation tests alone could overestimate primary TMDs when comorbid conditions are present.

Clinical relevance: Dynamic/static tests should be used as part of the routine TMD assessment. In case of Lyme disease as the actual cause of the facial pain, while the dentist might be suspecting TMD when dynamic/static TMD tests are negative, referral to an appropriate specialist for the diagnosis and treatment of Lyme disease needs to be made.
\end{abstract}

Keywords Dynamic/static tests $\cdot$ Palpation tests $\cdot$ Differential diagnosis $\cdot$ Temporomandibular pain $\cdot$ Comorbidity $\cdot$ Lyme disease

\section{Introduction}

Lyme disease, also known as borreliosis, is a multiorgan animal-borne disease, caused by bacteria — spirochetes of the

Magdalena Osiewicz

Magdalena.osiewicz@uj.edu.pl

1 Department of Integrated Dentistry, Dental Institute, Faculty of Medicine, Jagiellonian University Medical College, Krakow, Poland

2 School of Dentistry, University of Siena, Siena, Italy

3 Department of Infectious and Tropical Diseases, Faculty of Medicine, Jagiellonian University Medical College, Krakow, Poland

4 Department of Orofacial Pain and Dysfunction, Academic Centre for Dentistry Amsterdam (ACTA), University of Amsterdam and Vrije Universiteit Amsterdam, Amsterdam, The Netherlands
Borrelia species, classified as Borrelia burgdorferi $(\mathrm{Bb})$ strain [1]. It is the most common tick-borne infectious disease in Europe [2]. The number of people testing positive for anti$\mathrm{Bb}$ antibodies varies in different European countries (approximately 5-25\%) [3]. The Asbrink and Hovmark classification defines two stages of the disease: early and late Lyme disease [4]. Erythema migrans (EM), a pathognomic skin lesion that occurs in approximately $80 \%$ of Lyme disease patients, is the first symptom of early Lyme disease [3, 5]. In patients without EM or not undergoing treatment, the infection may spread and affect various organs. If the nervous system is infected, Lyme neuroborreliosis is diagnosed (LNB), whilst and if the infection affects the joints, Lyme arthritis is diagnosed (LA). Late Lyme disease is very rare and only occurs in patients who were not treated early enough. With the exception of the above-described early changes in the patient's skin, signs and symptoms are not characteristic of the disease, and blood 
culture to search for specific antibodies against $\mathrm{Bb}$ is needed for diagnostic purposes. In the very early stages, antibodies may not be present, and unspecific symptoms, such as pain in the facial area, are hard to relate to the presence of Lyme disease.

This means that early symptoms of Lyme disease may resemble, amongst others, the fluctuating nature of temporomandibular disorders (TMDs), such as brief periods of pain in the temporomandibular joint (TMJ) or masticatory muscles that may be followed by periods of complete remission [6]. Limitation of mouth opening may also occur $[7,8]$. Thus, patients who actually suffer from facial pain due to Lyme disease may be wrongly diagnosed with TMD. Orofacial pain has a prevalence of about $10 \%$ in the general population, and many conditions share similar clinical features [9]. TMD occurrence has been reported in patients with some chronic pain conditions (e.g., fibromyalgia, whiplash-associated disorders) or psychological disorders (e.g., depression or somatization) [10-14]. On the other hand, it needs to be pointed out that there are no available data on the frequency of TMD-pain diagnoses in patients with Lyme disease.

A TMD-pain diagnosis is commonly based on oral history and the outcomes of palpation tests as per the Research Diagnostic Criteria for TMD (RDC/TMD; [15]), or the updated Diagnostic Criteria for TMD (DC/TMD; [16]). As a complimentary diagnostic option, dynamic/static tests have been proposed, based on the rationale to elicit pain in a function-dependent manner (i.e., as a result of movements of the jaw and static muscle efforts) [17-20]. In patients with TMD symptoms occurring in presence of other primary conditions, such as Lyme disease, it should be interesting to assess findings from the dynamic/static tests with respect to the possible overestimation of primary TMDs that could be drawn from palpation tests alone.

Based on the above, this study has been performed in a population of patients with Lyme disease with the following twofold aim: (1) to determine the frequency of RDC/TMDbased pain diagnoses and dynamic/static tests-based pain diagnoses; and (2) to assess the agreement of palpation tests with static/dynamic tests in this population.

\section{Materials and methods}

\section{Participants}

The study was conducted at the Department of Infectious and Tropical Diseases of the University Hospital in Krakow on 86 consecutive adult patients with Lyme disease. Inclusion in the study was confined to individuals with confirmed Lyme arthritis or Lyme neuroborreliosis diagnoses, without any other acute or chronic inflammatory or systemic diseases that might have affected the presence of TMD symptoms, and without a history of TMD pain and/or dental pain. Since in this study the agreement of clinical TMD pain tests was determined, these tests were not used to exclude participants from the study. Thus, the oral history was used as the approach to rule out TMD pain [19].

Lyme disease was diagnosed according to the specific criteria that are fully described in the recommendations of The Polish Society of Epidemiologists and Infectious Diseases Doctors [3, 5].

The patients were divided into four groups: early Lyme arthritis (ELA), late Lyme arthritis (LLA), early Lyme neuroborreliosis (ELN), and late Lyme neuroborreliosis (LLN) $[3,5]$.

ELA/LLA was diagnosed in patients with symptoms of arthritis and the presence of positive antibodies against $\mathrm{Bb}$ class IgM or class IgG in the blood serum. ELN/LLN was diagnosed in patients showing symptoms of central or peripheral nervous system involvement and the presence of positive antibodies against $\mathrm{Bb}$ class IgM or class IgG in the blood serum and cerebrospinal fluid. Discrimination between ELA and LLA as well as between ELN and LLN depends on the time frame of symptoms' persistence.

The study was conducted according to the principles of the Helsinki declaration and approved by the Bioethics Committee of the Jagiellonian University (No. KBET/200/B/2011).

\section{Study design}

During a dedicated session at the Department of Infectious and Tropical Diseases, a specialist in TMD performed an examination according to the Research Diagnostic Criteria for Temporomandibular Disorders (RDC/TMD) guidelines [15], and the dynamic/static tests of the masticatory system [17, 18]. The examiner was trained by a calibrated RDC/TMD examiner (FL) within the framework of a 3-year specialty program in TMD and Orofacial Pain at the Department of Oral Kinesiology, Academic Centre for Dentistry Amsterdam (ACTA), Amsterdam, The Netherlands [21]. Furthermore, the patients were asked to complete the Polish version of the RDC/TMD questionnaire [22, 23].

The RDC/TMD examination included all clinical tests needed for an Axis I pain diagnosis. Palpation was applied in 12 different locations, on the left side as well as on the right side of the face and head, in the posterior, middle, and anterior temporalis muscle; the origin, body, and insertion of masseter muscle; the postmandibular region; the submandibular region; the lateral pterygoid area; the tendon of temporalis; and the lateral aspect of the TMJ and the posterior aspect of the TMJ. The patients' pain was scored on a 4-point ordinal pain scale: $0=$ no pain, $1=$ mild pain, $2=$ moderate pain, and $3=$ severe pain. Based upon the clinical measurements derived from the clinical RDC/TMD examination, one or more of the following TMD pain diagnoses could be established myofacial pain, 
myofascial pain with limited mouth opening, arthralgia, and osteoarthritis [15].

After the RDC/TMD examination, the dynamic and static tests were performed. These tests aim to provoke pain in the temporomandibular joints and/or masticatory muscles through loaded movements of the mandible and heavy static muscular effort, respectively.

During the dynamic tests, the patient was asked to perform jaw movements (i.e., mouth opening, closing, protrusion), while the examiner applied a slight manual counter pressure to the mandible ( $\pm 5 \mathrm{~N}$, as practiced on a weight scale). During the static tests, the patient was not allowed to move the mandible at all; it remained in a position approximately $5 \mathrm{~mm}$ in the direction of the intended movement, while the examiner gradually increased the manual resistance to jaw movements to impede them. The condition of maximum force exerted by the patient under resistance was maintained for $3 \mathrm{~s}$. During the opening and closing test, the mandible was held at a mouth opening of about $10 \mathrm{~mm}$, as measured interincisally. During the protrusion tests, the jaw was kept in a slightly forward position, viz., about $5 \mathrm{~mm}$ without tooth contact [17]. Following each test of this type (dynamic or static), pain scores were assessed with the same strategy as for the palpation tests [18].

\section{Data analysis}

For RDC/TMD-based pain diagnoses and dynamic/static tests-based pain diagnoses, descriptive frequencies were calculated.

In order to perform data analysis, the 4-point ordinal pain scale was dichotomized into: $0=$ no pain or $1=$ presence of pain (independent of the rating). The pain diagnoses based on palpation tests (RDC/TMD) were compared with those based on dynamic/static tests as per the study of Osiewicz et al. [20]. To that aim, the following single diagnostic outcomes were established for each diagnostic system (i.e., palpation tests and dynamic/static tests): no pain diagnosis, mainly myogenous pain, and mainly arthogenous pain.

The RDC/TMD diagnosis was based on the sum of all the points according to the dichotomized pain scale - the sum of the points divided by the number of palpation sites used for that summation (normalized sum). If the normalized sum of points for muscle palpation (excluding intra-oral sites) was greater than that for joint palpation, mainly myogenous pain was diagnosed. If the normalized sum of points for joint palpation was greater than that of muscle palpation, this indicated the presence of a mainly arthogenous pain. When the normalized sum of points for the palpation of the muscles and the joints were identical, the diagnosis was mainly myogenous pain.

As far as the dynamic/static tests were concerned, if the normalized sum of points for the static tests was greater than the sum obtained in the course of dynamic tests, a mainly myogenous pain was diagnosed. In the inverse situation (i.e., higher normalized sum of points for dynamic testing than for static testing), mainly arthogenous pain was diagnosed. If the normalized sums of points for dynamic testing and static testing were identical, a mainly myogenous pain was diagnosed.

Chi-square test was used to assess the difference between the two tests for the frequency of palpation-based diagnoses and of dynamic/static-based diagnoses in Lyme disease diagnoses.

Kappa statistic was used to assess the agreement between pain diagnoses based on palpation tests versus dynamic/static tests, and was interpreted according to Landis and Koch (1977): $0=$ poor, $0.01-0.20=$ slight, $0.21-0.40=$ fair, $0.41-$ $0.60=$ moderate, $0.61-0.80=$ substantial, and $0.81-1=$ al most perfect.

Statistics was performed using the StatsDirect version 2.8.0.

\section{Results}

The mean $( \pm \mathrm{SD})$ age of the study sample was $57.0( \pm 14.3)$ years, and the male/female ratio was $42 / 44$. As for specific Lyme disease diagnoses, ELA was confirmed in 14 patients, LLA was confirmed in 28 patients, ELN was confirmed in 33 patients, and LLN was confirmed in 11 patients.

The number of patients and frequency of RDC/TMD-based pain diagnoses and dynamic/static tests-based pain diagnoses are presented in Table 1 .

Mainly myogenous pain based upon palpation tests was diagnosed in 61 patients $(70.9 \%)$, mainly myogenous pain based on dynamic/static tests was diagnosed in 6 patients (7\%), mainly arthogenous pain based on dynamic/static tests was diagnosed in 4 patients (4.6\%), while mainly arthogenous pain based on palpation tests was not diagnosed in any patient.

Table 1 Number of patients and frequency of palpation-based pain diagnoses and dynamic/static tests-based pain diagnoses

\begin{tabular}{lll}
\hline Variable & $\begin{array}{l}\text { Number of } \\
\text { patients }\end{array}$ & $\begin{array}{l}\text { Frequency } \% \\
(N=86)\end{array}$ \\
\hline $\begin{array}{l}\text { RDC/TMD } \\
\text { Myofascial pain }\end{array}$ & 58 & $67.5 \%$ \\
$\quad \begin{array}{l}\text { Myofascial pain with } \\
\quad \text { limited mouth opening }\end{array}$ & 3 & $3.5 \%$ \\
$\quad \begin{array}{l}\text { Arthralgia } \\
\text { Osteoarthritis }\end{array}$ & 7 & $8.1 \%$ \\
Dynamic/static tests & 4 & $4.6 \%$ \\
$\quad$ Mainly myogenous pain & 6 & $7 \%$ \\
$\quad$ Mainly arthrogenous pain & 5 & $5.8 \%$ \\
No diagnosis & 3 & $3.5 \%$ \\
\hline
\end{tabular}


Differences in the frequency of diagnoses between the two methods were statistically significant $(p<0.01)$, with the exception of mainly arthogenous pain $(p=0.129)$.

As for mainly myogenous pain, differences between the number of patients with palpation-based and dynamic/static tests-based diagnoses were significant in all four groups of individuals with Lyme disease, i.e., for ELA $(p<0.001)$, LLA $(p<0.001)$, ELN $(p<0.001)$, and LLN $(p<0.001)$ (Table 2$)$.

The kappa value between the two diagnostic strategies was 0.06 , which is qualified as "poor."

\section{Discussion}

The prevalence of TMD signs and symptoms has been reported in patients affected by several comorbidities, such as chronic pain or psychological disorders $[10,12]$. Amongst those, there are no available data on the frequency of TMD-pain diagnoses in patients with Lyme disease, a condition of which the patient is often unaware and that could present symptoms mimicking TMDs. Based on that, the aims of this study were to assess the frequency of TMD-pain diagnoses in a population of individuals with Lyme disease and to establish how the diagnosis is influenced by the examination approach that is used (i.e., standard RDC/TMD palpation protocol vs dynamic/static tests).

The absence of data on TMD symptoms in Lyme disease renders it impossible to compare this study's findings with the available literature. On the other hand, they might be added to the amount of studies suggesting an increased frequency of TMD-like symptoms in patients with other primary conditions. For instance, the study of Gallotta et al. [12] showed patients with irritable bowel syndrome (IBS) have a higher risk of having TMD, whilst Grozdinska et al. [14] reported that the frequency of TMD muscle pain diagnoses was three times higher in patients with Hashimoto thyroiditis with respect to a control group of healthy individuals.

Within these premises, findings from the present investigation should alert clinicians on the fact that, if the diagnosis was based on palpation alone and the patients did not know if they had Lyme or not, $70 \%$ of them were diagnosed with RDC/ TMD myofascial pain. Thus, using dynamic/static tests in addition to palpation tests can help avoiding a potential overestimation of primary TMDs when comorbid conditions are present. The results of this study could support the suggestion of Visscher et al. that the dynamic/static tests yield less false positives than the outcomes of palpation tests [19].

A test for the recognition of TMD pain should only be theoretically able to discriminate between the patients' local musculoskeletal pain complaint and comorbid factors, which may cause central sensitization or widespread pain, considering that this study seems to suggest that palpation tests are potentially related to a generalized hypersensitivity, and not only to local musculoskeletal complaints [24, 25]. Hypersensitivity is caused by neurophysiological mechanisms, with a combination of an increased pain sensitivity and/or a reduced pain inhibition, leading up to a lowered pain threshold. This, in turn, may explain patients' pain evoked with palpation tests.

In the present investigation, it could be speculated that Lyme disease influenced both the outcomes of palpation tests and of dynamic/static tests, and that this influence is especially relevant for muscle palpation tests. These findings are hard to compare with the existing literature due to the paucity of publications on the topic. The study of Lorduy et al. provided preliminary evidence that the symptoms of central sensitization are more pronounced amongst individuals with an Axis I group I muscle disorder, in comparison with the other two types of TMD disorders based on palpation tests [26]. In line with those observations, the present study suggests that palpation tests may tend to overestimate the frequency of muscle

Table 2 Frequency $(\%)$ and number of patients $(N=86)$ distribution of palpation-based diagnoses and of dynamic/static-based diagnoses in Lyme disease diagnoses. NS, not significant

\begin{tabular}{|c|c|c|c|c|c|c|}
\hline \multirow[t]{2}{*}{ Lyme disease } & \multirow[t]{2}{*}{ Pain } & \multicolumn{2}{|c|}{ Palpation } & \multicolumn{2}{|c|}{ Dynamic/static } & \multirow[t]{2}{*}{$p$ (chi-square test) } \\
\hline & & $N$ & $\%$ & $N$ & $\%$ & \\
\hline \multirow[t]{2}{*}{$\operatorname{ELA}(n=14)$} & Mainly myogenous pain & 8 & $57.1 \%$ & 1 & $7.1 \%$ & $p<0.001$ \\
\hline & Mainly arthogenous pain & 0 & $0.0 \%$ & 0 & $0.0 \%$ & NS \\
\hline \multirow[t]{2}{*}{ LLA $(n=28)$} & Mainly myogenous pain & 18 & $64.3 \%$ & 3 & $10.7 \%$ & $p<0.001$ \\
\hline & Mainly arthogenous pain & 0 & $0.0 \%$ & 0 & $0.0 \%$ & NS \\
\hline \multirow[t]{2}{*}{$\operatorname{ELN}(n=33)$} & Mainly myogenous pain & 27 & $81.8 \%$ & 2 & $6.1 \%$ & $p<0.001$ \\
\hline & Mainly arthogenous pain & 0 & $0.0 \%$ & 4 & $12.1 \%$ & $p=0.12$ \\
\hline \multirow[t]{2}{*}{$\operatorname{LLN}(n=11)$} & Mainly myogenous pain & 8 & $72.7 \%$ & 0 & $0.0 \%$ & $p<0.001$ \\
\hline & Mainly arthogenous pain & 0 & $0.0 \%$ & 0 & $0.0 \%$ & $\mathrm{NS}$ \\
\hline \multirow{2}{*}{$\begin{array}{l}\text { All study participants } \\
\text { with Lyme disease }(n=86)\end{array}$} & Mainly myogenous pain & 61 & $70.9 \%$ & 6 & $7.0 \%$ & $p<0.001$ \\
\hline & Mainly arthogenous pain & 0 & $0.0 \%$ & 4 & $7.0 \%$ & $p=0.13$ \\
\hline
\end{tabular}


disorders in patients with a primary diagnosis of Lyme disease, which as a comorbid condition that can cause widespread muscle sensitization.

The study focused on four types of Lyme disease, depending on the site of pain and stage of disease. In Lyme neuroborreliosis, the presence of facial pain is a consequence of nerve involvement, whereas in Lyme arthritis the pain is the result of joint structure inflammation. Moreover, the stage of the inflammation - early or late - may have some influence on the presence of TMD-like symptoms. However, a poor correlation between palpation tests and dynamic/static tests was found in all the sub-groups of Lyme disease, thus suggesting that $\mathrm{Bb}$ infection per se, regardless of the clinical picture and stage of the disease, may influence the result of palpation tests.

There are several factors that might have affected the outcomes of this investigation. The main problem in this kind of study is that the results of the clinical examination cannot be used for the recognition of TMD pain in patients to avoid circularity. That is why establishing whether the patient was actually suffering from TMD pain or not was based on oral history. Any other potential causes of the pain were excluded by other medical specialists in the field. Another critical issue is that dynamic/ static tests are not used as standard to diagnose TMD pain yet, and this is why a suitable training in the specific techniques and in the verbal instructions to the patients is necessary. As a recommendation for future studies, it could be interesting to add an evaluation of the psychosocial status of the patients with Lyme disease, for an assessment of its possible influence on the agreement of the two diagnostic strategies for TMD pain. In addition, the adoption of the updated DC/TMD version, which was not available at the time of this study design, might help gathering data for comparing these findings (16).

On the other hand, the strength of this study is that, for the first time, it assessed the frequency of TMD symptoms in one of the most common and important infectious diseases in the northern hemisphere, which is Lyme disease.

In conclusion, the results of the study suggest that dynamic/ static tests should be used as part of the routine TMD assessment. In this investigation, they yield better results in case of Lyme disease as the actual cause of the facial pain, while the dentist might be suspecting TMD. So, when the dynamic/ static TMD tests are negative in such cases, referral to an appropriate specialist for the diagnosis and treatment of Lyme disease can be made. In this way, intervention delays and/or unnecessary treatments can be avoided.

\section{Conclusions}

Findings from the present investigation in a population of subjects with Lyme disease suggest that $70 \%$ of them were positive for a RDC/TMD diagnosis of myofascial pain.
Complementing the RDC/TMD assessment protocol with static/dynamic test led to a poor agreement between the two diagnostic strategies. Based on that, it might be suggested that palpation tests tend to overestimate the frequency of muscle disorders in patients with a primary diagnosis of Lyme disease, possibly due to the fact that the presence of comorbid conditions cause widespread muscle sensitization.

\section{Compliance with ethical standards}

Conflict of interest The authors declare that they have no conflict of interest.

Ethical approval All procedures performed in studies involving human participants were in accordance with the ethical standards of the institutional and/or national research committee and with the 1964 Helsinki declaration and its later amendments or comparable ethical standards.

Informed consent Informed consent was obtained from all individual participants included in the study.

Open Access This article is distributed under the terms of the Creative Commons Attribution 4.0 International License (http:// creativecommons.org/licenses/by/4.0/), which permits unrestricted use, distribution, and reproduction in any medium, provided you give appropriate credit to the original author(s) and the source, provide a link to the Creative Commons license, and indicate if changes were made.

\section{References}

1. Stanek G, Wormser GP, Gray J, Strle F (2012) Lyme borreliosis. Lancet (London, England) 379:461-473. https://doi.org/10.1016/ S0140-6736(11)60103-7

2. Derdáková M, Lencáková D (2005) Association of genetic variability within the Borrelia burgdorferi sensu lato with the ecology, epidemiology of Lyme borreliosis in Europe. Ann Agric Environ Med 12:165-172

3. Biesiada G, Czepiel J, Leśniak MR, Garlicki A, Mach T (2012) Lyme disease: review. Arch Med Sci 8:978-982. https://doi.org/ 10.5114/aoms.2012.30948

4. Asbrink E, Hovmark A (1993) Classification, geographic variations, and epidemiology of Lyme borreliosis. Clin Dermatol 11: 353-357

5. Pancewicz SA, Garlicki AM, Moniuszko-Malinowska A, Ziajkowska J, Kondrusik M, Grygorczuk S, Czupryna P, Dunaj J (2015) Diagnostyka i leczenie chorób przenoszonych przez kleszcze. Rekomendacje polskiego towarzystwa epidemiologów i lekarzy chorób zakaźnych. Przegl Epidemiol 69:421-428

6. Heir GM, Fein LA (1996) Lyme disease: considerations for dentistry. J Orofac Pain 10:74-86

7. Wolańska-Klimkiewicz E, Szymańska J, Bachanek T (2010) Orofacial symptoms related to boreliosis-case report. Ann Agric Environ Med 17:319-321

8. te Veldhuis E, Lobbezoo F, te Veldhuis A et al (2011) Residual orofacial complaints following Lyme neuroborreliosis: an unusual case of TMD. J Craniomandib Funct 3:221-228

9. Dworkin SF, Massoth DL (1994) Temporomandibular disorders and chronic pain: disease or illness? J Prosthet Dent 72:29-38

10. Bertoli E, de Leeuw R (2016) Prevalence of suicidal ideation, depression, and anxiety in chronic temporomandibular disorder 
patients. J Oral Facial Pain Headache 30:296-301. https://doi.org/ 10.11607/ofph.1675

11. Costa YM, Conti PCR, de Faria FAC, Bonjardim LR (2017) Temporomandibular disorders and painful comorbidities: clinical association and underlying mechanisms. Oral Surg Oral Med Oral Pathol Oral Radiol 123:288-297. https://doi.org/10.1016/j.oooo. 2016.12.005

12. Gallotta S, Bruno V, Catapano S, Mobilio N, Ciacci C, Iovino P (2017) High risk of temporomandibular disorder in irritable bowel syndrome: is there a correlation with greater illness severity? World J Gastroenterol 23:103-109. https://doi.org/10.3748/wjg.v23.i1. 103

13. Landzberg G, El-Rabbany M, Klasser GD, Epstein JB (2017) Temporomandibular disorders and whiplash injury: a narrative review. Oral Surg Oral Med Oral Pathol Oral Radiol 124:e37-e46. https://doi.org/10.1016/j.oooo.2017.03.001

14. Grozdinska A, Hofmann E, Schmid M, Hirschfelder U (2018) Prevalence of temporomandibular disorders in patients with Hashimoto thyroiditis. J Orofac Orthop/Fortschritte der Kieferorthopädie 79:277-288. https://doi.org/10.1007/s00056018-0140-6

15. Dworkin SF, LeResche L (1992) Research diagnostic criteria for temporomandibular disorders: review, criteria, examinations and specifications, critique. J Craniomandib Disord 6:301-355

16. Schiffman E, Ohrbach R, Truelove E, Look J, Anderson G, Goulet JP, List T, Svensson P, Gonzalez Y, Lobbezoo F, Michelotti A, Brooks SL, Ceusters W, Drangsholt M, Ettlin D, Gaul C, Goldberg LJ, Haythornthwaite JA, Hollender L, Maixner W, van der Meulen M, Murray GM, Nixdorf DR, Palla S, Petersson A, Pionchon P, Smith B, Visscher CM, Zakrzewska J, Dworkin SF (2014) Diagnostic criteria for temporomandibular disorders (DC/ TMD) for clinical and research applications: recommendations of the international RDC/TMD consortium network* and orofacial pain special interest group $\dagger$. J Oral Facial Pain Headache 28:627. https://doi.org/10.11607/jop.1151

17. Naeije M, Hansson TL (1986) Electromyographic screening of myogenous and arthrogenous TMJ dysfunction patients. J Oral Rehabil 13:433-441
18. Visscher CM, Lobbezoo F, Naeije M (2007) A reliability study of dynamic and static pain tests in temporomandibular disorder patients. J Orofac Pain 21:39-45

19. Visscher CM, Naeije M, De Laat A et al (2009) Diagnostic accuracy of temporomandibular disorder pain tests: a multicenter study. J Orofac Pain 23:108-114

20. Osiewicz MA, Manfredini D, Loster BW, van Selms MKA, Lobbezoo F (2018) Comparison of the outcomes of dynamic/ static tests and palpation tests in TMD-pain patients. J Oral Rehabil 45:185-190. https://doi.org/10.1111/joor.12600

21. Lobbezoo F, van der Zaag J, Visscher CM, Naeije M (2004) Oral kinesiology. A new postgraduate programme in the Netherlands. J Oral Rehabil 31:192-198

22. Osiewicz MA, Lobbezoo F, Loster BW, Wilkosz M, Naeije M, Ohrbach R (2010) Badawcze kryteria diagnostyczne zaburzeń czynnościowych układu ruchowego narządu żucia BKD/ ZCURNŻ — polska wersja dwuosiowego systemu diagnostycznego ZCURNŻ. Protet Stomatol 60:433-444

23. Osiewicz MA, Lobbezoo F, Loster BW, Wilkosz M, Naeije MOR (2013) Badawcze Kryteria Diagnostyczne Zaburzeń Czynnościowych Układu Ruchowego Narządu Żucia BKD/ ZCURNŻ - polska wersja dwuosiowego systemu diagnostycznego ZCURNŻ. J Stomatol 66:576-649

24. Diatchenko L, Nackley AG, Slade GD, Fillingim RB, Maixner W (2006) Idiopathic pain disorders-pathways of vulnerability. Pain 123:226-230. https://doi.org/10.1016/j.pain.2006.04.015

25. Koutris M, Visscher CM, Lobbezoo F, Naeije M (2013) Comorbidity negatively influences the outcomes of diagnostic tests for musculoskeletal pain in the orofacial region. Pain 154:927-932. https://doi.org/10.1016/j.pain.2013.03.004

26. Lorduy KM, Liegey-Dougall A, Haggard R, Sanders CN, Gatchel RJ (2013) The prevalence of comorbid symptoms of central sensitization syndrome among three different groups of temporomandibular disorder patients. Pain Pract 13:604-613. https://doi.org/10.1111/papr.12029

Publisher's note Springer Nature remains neutral with regard to jurisdictional claims in published maps and institutional affiliations. 